\title{
CLIA '88 lab regulations are watered down
}

The current FORT COLLINS, Colo.-In the summer of 1988, news reports and congressional testimony painted an alarming picture: untrained laboratory personnel were performing complex diagnostic tests in unregulated laboratories and offices around the country. As a result, Congress passed the Clinical Laboratory Improvements Amendments of 1988 (CLIA '88), legislation to regulate who could perform what tests in each of the nation's 350,000 clinical laboratory facilities, ranging from the most sophisticated hospital laboratories to microscope-and-centrifuge set-ups in doctor's offices.

The American Society for Microbiology (ASM, Washington, DC), the American Society of Clinical Pathologists (Washington, DC), and other trade associations representing highly trained laboratory personnel lobbied hard for stringent regulations. "Only certified technologists are adequately trained to make the decisions required to deliver quality laboratory diagnoses of infectious diseases," says Alice Weissfeld, a member of ASM's Public and Scientific Affairs Advisory Board.

The American Medical Association (Chicago, IL) countered that strict training requirements would require most physicians to dismiss their current laboratory employees or pay for expensive retraining programs. This would result in closings of small laboratories, which would cause medical costs to skyrocket as physicians were forced to rely on private clinical laboratories.

It appears that physicians have won this battle. Today, nearly five years after CLIA ' 88 became law, the Department of Health and $\mathrm{Hu}$ man Services is still struggling to publish the final rule that will spell out exactly which tests will be most regulated and how it will enforce the act. Nevertheless, the most re-

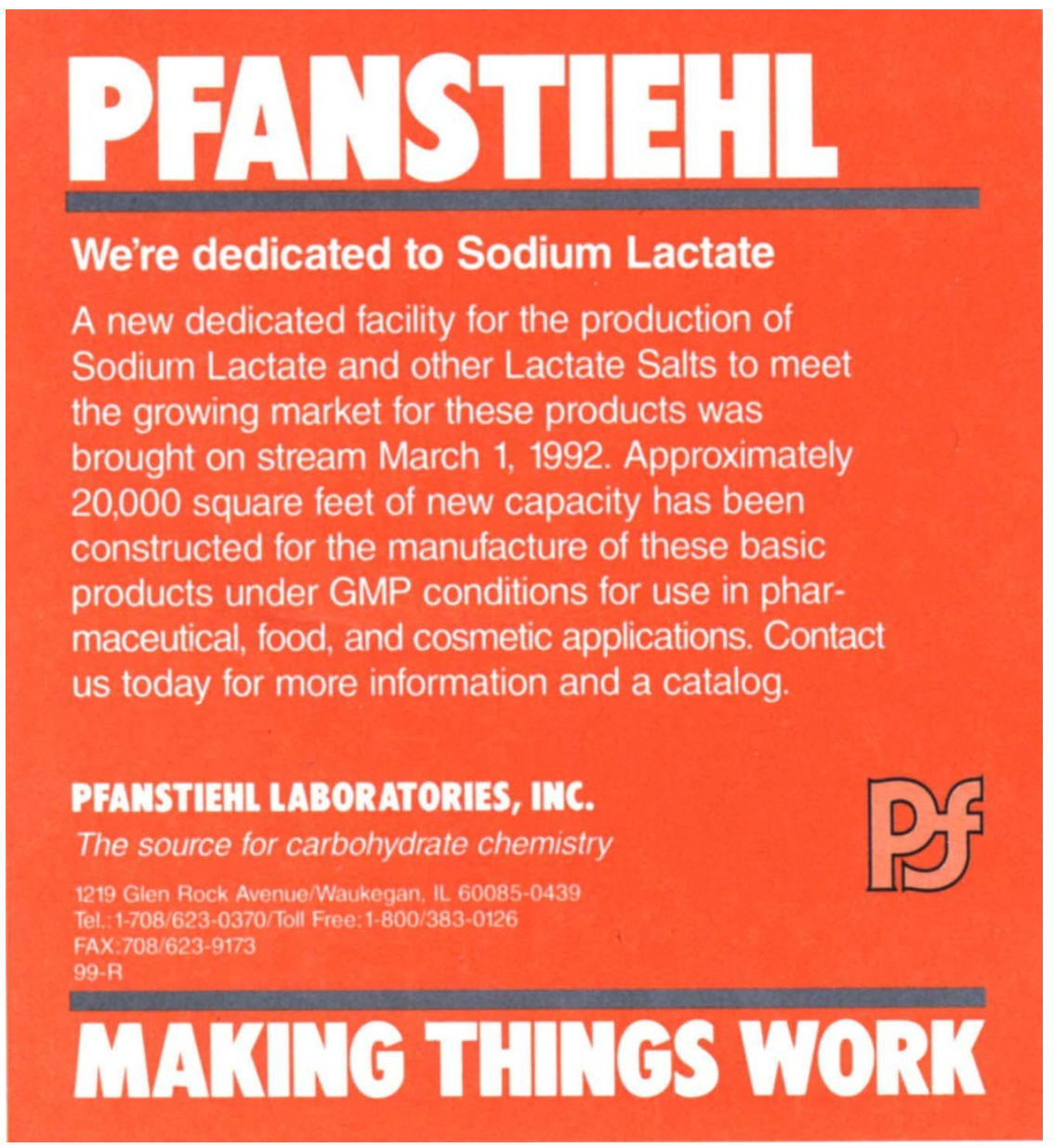

Write in No. 538 on Reader Service Card

cent version, published in January, allows physicians to continue business with those employees already working in their laboratories. New laboratory personnel will have to meet increased training requirements.

Still unclear is what steps companies will have to take to have new diagnostic tests classified under the provisions of CLIA ' 88 , though the Food and Drug Administration (Bethesda, MD) will oversee the process. The regulations spell out three classes of tests: exempt, moderately complex, and complex. Simple, one-step tests--such as Quidel's (San Diego, CA) new inoffice pregnancy test-fall into the exempt category. Anyone can perform these tests, and no supervision is required.

About 75 percent of the tests already classified fall into the moderately complex category, including monoclonal-antibody-based tests for sexually transmitted diseases of nonviral origin. Personnel performing moderately complex tests will have to be high-school graduates with "documented training" for specific tests.

The remaining tests, including most microbiology and pathology diagnostics, fall into the highly complex category. Test personnel must have at least an associate degree plus "further training." For all categories, however, current clinicallaboratory personnel are grandfathered until 1997, by which time they will have to meet the more stringent training requirements.

No one is certain what the final effect of the regulations will be on those companies developing diagnostics. On the one hand, the current watered-down regulations make it likely that new products will not be shut out of any market, whether it be physician offices or large clinical laboratories. But more stringent regulations could actually benefit diagnostic companies by raising personnel standards. Said one company official, "It would be much easier for us to design a diagnostic if we could be sure that the lab personnel using it were highly trained. As it is, we try to design a test for the lowest common denominator."

-Joseph Alper 